\title{
Extreme Value Analysis of Statistically Independent Stochastic Variables
}

\author{
Yongho Choi $\circledast^{*}$, Seong Mo Yeon $\circledast^{*}$, Hyunjoe Kim $\circledast^{*}$ and Dongyeon Lee $\circledast^{*}$ \\ "Ship \& Offshore Research Center, Samsung Heavy Industries Co. Ltd., Daejeon, Korea
}

KEY WORDS: EVA (extreme value analysis), EVT (extreme value theory), IID (independent and identically distributed), Weibull, GPD (generalized Pareto distribution), Block maxima, GEV (generalized extreme value), POT (peaks-over-threshold), PACF (partial autocorrelation function)

\begin{abstract}
An extreme value analysis (EVA) is essential to obtain a design value for highly nonlinear variables such as long-term environmental data for wind and waves, and slamming or sloshing impact pressures. According to the extreme value theory (EVT), the extreme value distribution is derived by multiplying the initial cumulative distribution functions for independent and identically distributed (IID) random variables. However, in the position mooring of DNVGL, the sampled global maxima of the mooring line tension are assumed to be IID stochastic variables without checking their independence. The ITTC Recommended Procedures and Guidelines for Sloshing Model Tests never deal with the independence of the sampling data. Hence, a design value estimated without the IID check would be under-or over-estimated because of considering observations far away from a Weibull or generalized Pareto distribution (GPD) as outliers. In this study, the IID sampling data are first checked in an EVA. With no IID random variables, an automatic resampling scheme is recommended using the block maxima approach for a generalized extreme value (GEV) distribution and peaks-over-threshold (POT) approach for a GPD. A partial autocorrelation function (PACF) is used to check the IID variables. In this study, only one $5 \mathrm{~h}$ sample of sloshing test results was used for a feasibility study of the resampling IID variables approach. Based on this study, the resampling IID variables may reduce the number of outliers, and the statistically more appropriate design value could be achieved with independent samples.
\end{abstract}

\section{Abbreviations}
ACF: autocorrelation function
PACF: partial ACF
EVA: extreme value analysis
EVT: extreme value theory
GPD: generalized Pareto distr.
GEV: generalized extreme value
CI: confidence interval
MEF: mean excess function
IQR: interquartile range
SE: standard error
P-P: probability-probability
Q-Q: quartile-quartile
MPM: most probable maximum
MOM: method of moments
LS: least squares methods
MLE: maximum likelihood estimation method
PPC: Pearson correlation coefficient $=R$
IID: independent, identically distributed

\section{Introduction}

The extreme value theory (EVT) based on the Fisher-Tippett theorem (Fisher and Tippett, 1928) describes the distribution of extremes and rare events, especially in highly nonlinear slamming or sloshing impact data and long-term environmental applications. An inference about extremes is usually difficult to achieve because of the lack of data. In this case, the tail behavior of the distribution is of importance. There are mainly two popular extreme value models in the EVT: the block maximum model and peaks-over-threshold (POT) method. According to the 1st EVT theorem, which is referred to as the Fisher-Tippett-Gnedenko or Fisher-Tippett theorem, a distribution of the block maxima yields a generalized extreme value distribution. The 2nd EVT theorem, which is referred to as the Pickands-Balkemade Haan theorem (Balkema and Haan, 1974; Pickands, 1975), is used in the tail-fitting for the POT. The POT data also consist of two distributions: a Poisson distribution for the number of events per given time period, and a generalized Pareto distribution (GPD) for the size of the exceedances.

This paper will focus only on the EVT for the design value for the sloshing impact pressures obtained from a sloshing model test. It can be understandably applied to obtain a design environmental condition. In order to be able to apply the EVT, the observations need to be independent and identically distributed (IID). Hence, IID is to be checked for measured sloshing impact pressures. But DNVGL (2015) even describes that sampled global maxima of mooring line

Received 4 December 2018, revised 9 April 2019, accepted 13 June 2019

Corresponding author Yongho Choi: +82-42-865-4735, yh01.choi@samsung.com ORCID: https://orcid.org/0000-0002-6653-5476 It is noted that this paper is a revised edition based on the proceedings of the Fall Conference of KSOE 2018 in Incheon.

(C) 2019, The Korean Society of Ocean Engineers

This is an open access article distributed under the terms of the creative commons attribution non-commercial license (http://creativecommons.org/licenses/by-nc/3.0) which permits unrestricted non-commercial use, distribution, and reproduction in any medium, provided the original work is properly cited. 
tension are assumed to be independent stochastic variables without checking IID. ITTC (2017) never covers the independence of sampling pressures in a sloshing model test. Consequently, the assumption that consecutive data are IID may not be theoretically well justified, and the common positive correlation of successive data may lead to conservative estimates by simply regarding observations far away from a Weibull or generalized Pareto distribution as outliers.

This study first checked the IID of the sampled data. If random variables do not pass the IID check, resampling schemes that use the block maxima approach for the GEV (Generalized extreme value) and POT approach for the GPD are used. A partial autocorrelation function (PACF) is used to check automatically the IID variables. Finally, the resampled IID data are used to estimate the extreme design values. For the feasibility study of the resampling IID variables approach, only one $5 \mathrm{~h}$ sample of sloshing test results was used in this study.

\section{Theoretical Background}

\subsection{Extreme value theory}

Fisher and Tippett (1928) developed and organized a GEV using three asymptotic limit distributions: Gumbel $(\xi=0)$, Fréchet $(\xi>0)$, and Weibull $(\xi<0)$ distributions for IID variables (Ochi, 1990). Gnedenko (1943) and Gumbel (1954) gave the credit for the GEV or convergence to the type theorem (Choi 2016).

$$
G_{G E V}(x ; \mu, \sigma, \xi)= \begin{cases}\exp \left[-(1+\xi(x-\mu) / \sigma)^{-1 / \xi}\right] & \xi \neq 0 \\ \exp [-\exp (-(x-\mu) / \sigma)] & \xi=0\end{cases}
$$

in which $\mu, \sigma$, and $\xi$ represent the location ("Shift" in figures), scale, and shape parameters, respectively. The $r$-return period value $x_{r}$ is obtained by,

$$
x_{r}= \begin{cases}\hat{\mu}+\hat{\sigma} \hat{\xi}^{-1}\left[-\left(\ln \left(1-r^{-1}\right)\right)^{-\hat{\xi}}-1\right] & \xi \neq 0 \\ \hat{\mu}-\hat{\sigma} \ln \left(-\ln \left(1-r^{-1}\right)\right) & \xi=0\end{cases}
$$

The symbol ${ }^{\wedge}$ represents the estimation. For the sloshing test, the $r^{-}$return period in model scale for the $H r$-hour in real scale is obtained by $r=H r \times 3600 / d t / \sqrt{\lambda}=H r / D \times N_{p}$ and $d t=D \times 3600 /$ $N_{p} / \sqrt{\lambda}$, where $D, N_{p}, d t$, and $\lambda$ are the test duration in hours, number of peak pressures, average time interval of impacts in seconds, and real-to-model scale ratio, respectively.

The maximum likelihood estimation (MLE, see sec. 2.4) method is used to estimate the GEV parameters by maximizing the likelihood function $(L)$ or by minimizing the negative log-likelihood function $(-l)$.

$$
\begin{aligned}
& L(\mu, \sigma, \xi ; x)=\Pi_{i}^{n} g_{G E V}(x)=\Pi_{i}^{n} \frac{\partial G_{G E V}(x)}{\partial x} \\
& =\frac{1}{\sigma^{n}} \Pi_{i}^{n}\left[1+\xi \frac{x_{i}-\mu}{\sigma}\right]^{-\left(1+\frac{1}{\xi}\right)} \Pi_{i}^{n} \exp \left[-\left(1+\xi \frac{x_{i}-\mu}{\sigma}\right)^{-\frac{1}{\xi}}\right]
\end{aligned}
$$

$$
\begin{aligned}
& -l(\mu, \sigma, \xi ; x)=-\ln L(\mu, \sigma, \xi ; x) \\
& =n \ln \sigma+\left(1+\frac{1}{\xi}\right) \sum_{i}^{n} \ln \left[1+\xi \frac{x_{i}-\mu}{\sigma}\right]+\sum_{i}^{n}\left(1+\xi \frac{x_{i}-\mu}{\sigma}\right)^{-\frac{1}{\xi}}
\end{aligned}
$$

A Hessian matrix $H$ is defined to obtain the $S E$ (standard error $=s t d e v$ of sample mean $=s t d e v / \sqrt{N}$ ) for the $C I$ (confidence interval), in which stdev is a standard deviation. It is a square matrix of 2 nd order partial derivatives of a scalar-valued negative log-likelihood function. The estimation symbol ${ }^{\wedge}$ is dropped hereafter. An inverse matrix of $H$ yields the variance-covariance matrix $V$. The $S E$ S of the distribution parameters are obtained by the square root of the diagonal terms of $V$.

$$
V=H(-l)^{-1}, H(-l)=\left(\begin{array}{c}
-\frac{\partial^{2} l}{\partial \mu^{2}}-\frac{\partial^{2} l}{\partial \sigma \partial \mu}-\frac{\partial^{2} l}{\partial \xi \partial \mu} \\
-\frac{\partial^{2} l}{\partial \sigma \partial \mu}-\frac{\partial^{2} l}{\partial \sigma^{2}}-\frac{\partial^{2} l}{\partial \xi \partial \sigma} \\
-\frac{\partial^{2} l}{\partial \xi \partial \mu}-\frac{\partial^{2} l}{\partial \xi \partial \sigma}-\frac{\partial^{2} l}{\partial \xi^{2}}
\end{array}\right)
$$

Because the $S E$ s of the $r$-return period extreme values are more practical than the $S E \mathrm{~S}$ of the distribution, the Delta $(\nabla)$ method (Xu and Long, 2005) for the GEV is used. Hence, the $S E$ is the square root of the variance of the $r$-return period extreme value $\hat{x}_{r}$.

$$
\begin{aligned}
& S E_{\hat{x}_{r}}=\sqrt{\left(\operatorname{Var}\left(\hat{x}_{r}\right)\right.} \approx \sqrt{\nabla x_{r}^{T} \cdot V \cdot \nabla x_{r}}, y_{r}=-\ln \left(1-r^{-1}\right) \\
& \nabla x_{r}=\left(\begin{array}{c}
\frac{\partial x_{r}}{\partial \mu} \\
\frac{\partial x_{r}}{\partial \sigma} \\
\frac{\partial x_{r}}{\partial \xi}
\end{array}\right)=\left(\begin{array}{c}
1 \\
-\xi^{-1}\left(1-y_{r}^{-\xi}\right) \\
\sigma \xi^{-2}\left(1-y_{r}^{-\xi}\right)-\sigma \xi^{-1} y_{r}^{-\xi} \ln y_{r}
\end{array}\right)
\end{aligned}
$$

Next, Balkema and Haan (1974) and Pickands (1975) verified that the exceedances over a sufficiently large threshold value $u$ approximately yield the GPD when the IID variables follow a GEV distribution. The GPD is derived using the conditional exceedance distribution function for IID random variables.

$$
G_{G P D}(y)=\left\{\begin{array}{ll}
1-\left(1+\xi y / \sigma_{u}\right)^{-1 / \xi} & \xi \neq 0 \\
1-\exp \left(-y / \sigma_{u}\right) & \xi=0
\end{array}, \sigma_{u}=\sigma+\xi(u-\mu)\right.
$$

The $r$-return period value $x_{r}$ for the GPD is shown in the equation below. In this equation, $n_{y}$ is the number of measured points per unit time ( $1 \mathrm{~h}$ for the sloshing test, 1 year for the environment) and $\hat{\lambda}_{u=\operatorname{Pr}}(X>u)=1-F(u)$ is the 'Rate' (empirical threshold exceedance rate). In the case of sloshing tests, $r=r^{\prime} n_{y}=H r \times 3600 / d t / \sqrt{\lambda}$. In the case of the environment, $r=r^{\prime} n_{y}=r^{\prime} \times 365.25 \times 24 / \Delta$, where $r^{\prime}$ and $\triangle$ are the number of years and measuring interval in hours, respectively. 


$$
x_{r}= \begin{cases}u+\hat{\sigma} \hat{\xi}^{-1}\left[\left(r^{\prime} n_{y} \hat{\lambda}_{u}\right)^{-\hat{\xi}}-1\right] & \xi \neq 0 \\ u-\hat{\sigma} \ln \left(r^{\prime} n_{y} \hat{\lambda}_{u}\right) & \xi=0\end{cases}
$$

The negative log-likelihood function $(-l)$ for GPD is,

$$
-l(\mu, \sigma, \xi ; y)=n \ln \sigma+\left(1+\xi^{-1}\right) \cdot \sum_{i}^{n}\left(-\xi y_{i} / \sigma\right)
$$

The Delta method for the GPD of the $S E$ s for the $r$-return period extreme values is shown in the equation below. Hereafter, $t \leftarrow r^{\prime} n_{y}$, $\lambda \leftarrow \hat{\lambda}_{u}$.

$$
\nabla x_{r}=\left(\begin{array}{c}
\frac{\partial x_{r}}{\partial \mu} \\
\frac{\partial x_{r}}{\partial \sigma} \\
\frac{\partial x_{r}}{\partial \xi}
\end{array}\right)=\left(\begin{array}{c}
\sigma t(t \lambda)^{\xi-1} \\
\xi^{-1}\left[(t \lambda)^{\xi}-1\right] \\
-\sigma \xi^{-2}\left[(t \lambda)^{\xi}-1\right]+\sigma \xi^{-1}(t \lambda)^{\xi} \ln (t \lambda)
\end{array}\right)
$$

The determination of threshold $u$ is essential for the GPD because the POT approach provides a model for IID exceedances over a high threshold. There are various capable methods for determining $u$, such as the MEF (mean excess function or MRL (mean residual life), most popular method (Kim and Song, 2012)) plot, parameter stability plot, dispersion index plot, rule of thumb, and multiple-threshold model method (Bommier, 2014). Because the $M E F$ is a linear function of $u$, the starting point of linearity found in the MEF plot is to be a proper value as the threshold value.

$$
M E F=e(u)=E[X-u \mid X>u]=(\sigma+\xi u) /(1-\xi)
$$

\subsection{Other distributions}

In the conventional analysis procedures of the sloshing test to date, a 3-parameter Weibull distribution, which is one of the GEV distributions when $\xi<0$, or GPD is used to estimate the extreme design value. The 3-parameter Weibull for the highest $10 \%$ of the data as a popular industry practice was also studied. It is a hybrid approach with the POT and GEV instead of the GPD.

\subsection{Autocorrelation and partial autocorrelation}

The data for EVT should be IID stochastic variables. Because zero correlation shows that the variables are linearly independent, the independency is checked by the correlation. A coefficient of the autocorrelation function (ACF) $r_{x}(\tau)$ shows the correlation with lag $\tau$ between $x(t)$ and $x(t-\tau)$ in a given time series. The PACF $p_{x}(\tau, \tau)$ is defined by the autocorrelation between $x(t)$ and $x(t-\tau)$ in a given time series, with the linear dependence of $x(t)$ on $x(t-1), x(t-2), \cdots, x(t-\tau-1)$ removed.

$$
\begin{aligned}
& r_{x}(\tau)=\sum_{(t=1)}^{(n-\tau)}\left(x_{t}-\bar{x}\right)\left(x_{t+\tau}-\bar{x}\right) /\left(\sum_{t=1}^{n}\left(x_{t}-\bar{x}\right)^{2}\right. \\
& p_{x}(\tau, \tau)=\frac{\operatorname{cov}\left(x_{i}-x_{i-\tau} \mid x_{i-1}, \cdots, x_{i-\tau+1}\right)}{\sqrt{\operatorname{var}\left(x_{i} \mid x_{i-1}, \cdots, x_{i-\tau+1}\right) \cdot \operatorname{var}\left(x_{i-\tau} \mid x_{i-1}, \cdots, x_{i-\tau+1}\right)}}
\end{aligned}
$$

Where

$$
\left\{\begin{array}{cl}
p_{x}(1,1)=r_{x}(1) & \text { for } \tau=1 \\
p_{x}(\tau, \tau)=\frac{r_{x}(\tau)-\sum_{(j=1)}^{(\tau-1)} p_{x}(\tau-1, j) r_{x}(\tau-j)}{1-\sum_{(j-1)}^{(\tau-1)} p_{x}(\tau-1, j) r_{x}(j)} & \text { for } \tau=2,3, \cdots, n(15) \\
p_{x}(\tau, j)=p_{x}(\tau-1, j)-p_{x}(\tau, \tau) \cdot p_{x}(\tau-1, \tau-j) &
\end{array}\right.
$$

A program for all the statistical analyses except for $C I$ for sloshing impact pressures was realized in the Python programming language in this study, rather than the ' $R$ ' programming language and in-house code used in Choi (2016) for the extreme environmental condition.

\subsection{Parameter estimation methods of distribution}

When fitting probability distributions to data, DNVGL (2017) recommends several fitting techniques such as the method of moments (MOM), least squares methods (LS), and maximum likelihood estimation (MLE). MLE is used to fit the data on GEV and GPD. Because MLE for a Weibull is applicable only when the shape parameter is greater than one (Smith, 1985), and the shape parameter for the sloshing peak pressures is usually smaller than one, the MOM was applied for a Weibull in this study. Because the purpose of this study was to check the IID for statistical distribution models, a comparison of the fitting techniques was not carried out in this study.

\section{Sloshing Impact Pressures and Conventional Fitting}

Special care is needed to extract the sloshing impact pressures directly from the sloshing model test because of various causes of interference such as electronic noise and meaningless small pressures in the measured pressure time record. An initial POT method with an initial time window that is usually used in GTT is applied to cope with it (Fillon et al., 2011; Kim, 2017). Because the sloshing pressures sampled with this screening approach does not include the checking IID, an additional POT for GPD and block maxima for GEV for IID random variables were introduced in this study.

A sloshing model test with a model scale ratio $\lambda=50$ for the No. 2 tank of a $160 \mathrm{k}\left(160,000 \mathrm{~m}^{3}\right)$ conventional LNG carrier was conducted by filling it to $30 \%$ with water and air under 1 year North Atlantic beam sea condition. This study dealt with the sample pressure record from a single Kistler piezo-type pressure sensor at only one location. Fig. 1 shows the extracted $764 N_{p}$ sloshing peak pressures with an initial threshold of $2.5 \mathrm{kPa}$ and time window of $0.2 \mathrm{~s}$, which is hereafter defined as one block for the block maxima approach in the model scale. The measured maximum pressure was $244.7 \mathrm{kPa}$ in model scale. The total test duration was $2545.6 \mathrm{~s}$ in model scale $(D=5 h$ in real scale); $d t$ was $3.33 \mathrm{~s}$. Fig. 2 shows the results of the conventional procedure for the 3-parameter Weibull fitting with all 764 data points. The estimated $3 \mathrm{~h}$ MPM $(Q=1 / r=0.002)$ as a return level for the $3 \mathrm{~h}$ return period is $143.6 \mathrm{kPa}$, and it may be underestimated 


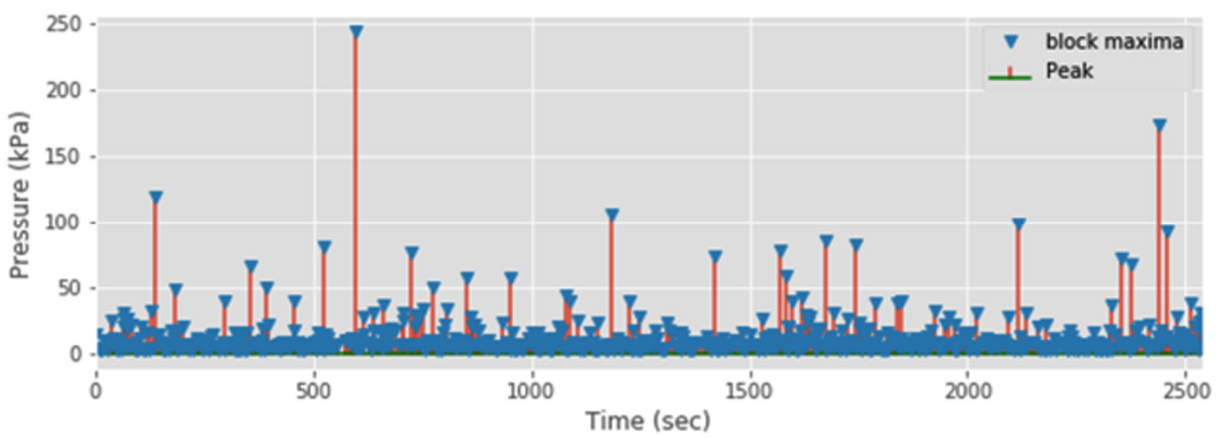

Fig. 1764 sloshing peak pressures (initial threshold of $2.5 \mathrm{kPa}$, time window of $0.2 \mathrm{~s}$ in model scale)

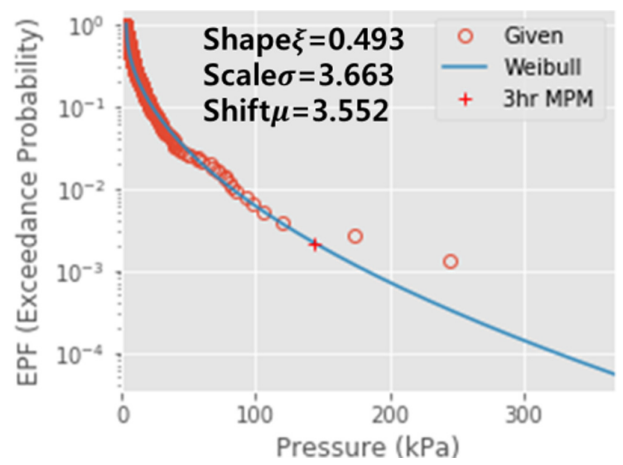

Fig. 2 3-parameter Weibull fitting with all 764 data points $(I Q R=$ $4.769, \gamma=5)$

considering the last two large pressures, where $r=3 h / 5 h \times 764=458.4$. A value of $143.6 \mathrm{kPa}$ (75.4 bar) for water yields $69.4 \mathrm{kPa}$ (35.4 bar) for $\mathrm{LNG}$ in real scale. The maximum value of the abscissa is obtained by multiplying the maximum measured data by 1.5 .

The Tukey (1977) method using quartiles is used to check the outliers. An outlier from the fitting curve is determined based on a distribution of residuals obtained by subtracting the theoretical values of a model's extreme distribution from samples with the assumption of normally distributed residuals. Then, the outsiders of $Q_{1}-\gamma \times I Q R$ to $Q_{3}+\gamma \times I Q R$ are defined as the outliers, where $Q_{1}, Q_{3}$, and $I Q R$ represent the first and third quartiles and a interquartile range $Q_{3}-Q_{1}$ of residuals, respectively. $\gamma$ is a constant value traditionally given as 1.5 by Tukey. However, in this paper, an appropriate value will be found, because smaller residuals yield a larger $\gamma$. In the case of Fig. 2 , the last two large pressures are considered as outliers with $I Q R=$
4.769 and $\gamma=5$.

Because the conventional procedure does not check the IID, no one makes sure whether or not the $3 \mathrm{~h}$ MPM of $143.6 \mathrm{kPa}$ is correct. To avoid this, the linear independence is checked by PACF, as shown in Fig. 3. Two graphical diagnostic plots such as the P-P plot and Q-Q plot, as shown in Fig. 4, are also used for the goodness of fit. In the Q-Q plot, R is the Pearson correlation coefficient (PCC), with a range of -1.0 to 1.0 .

Because one point at time lag $\tau=18$ of PACF in Fig. 3 exceeds the $95 \% C I$, and the plots in Fig. 4 look bad ( $\mathrm{R}=0.982$ in the $\mathrm{Q}-\mathrm{Q}$
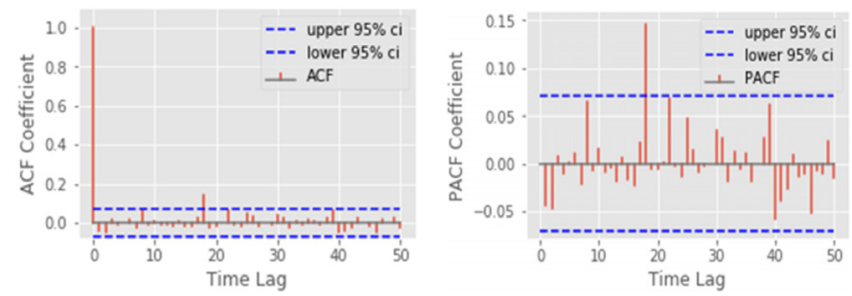

Fig. 3 ACF (left) and PACF (right) for all 764 data points
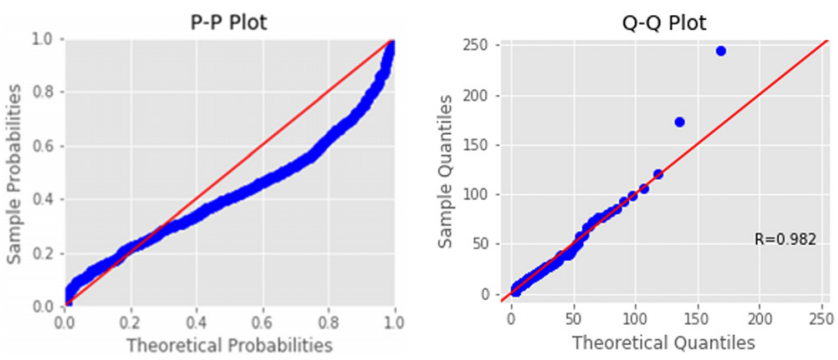

Fig. 4 P-P (left) and Q-Q (right) plots for all 764 data points

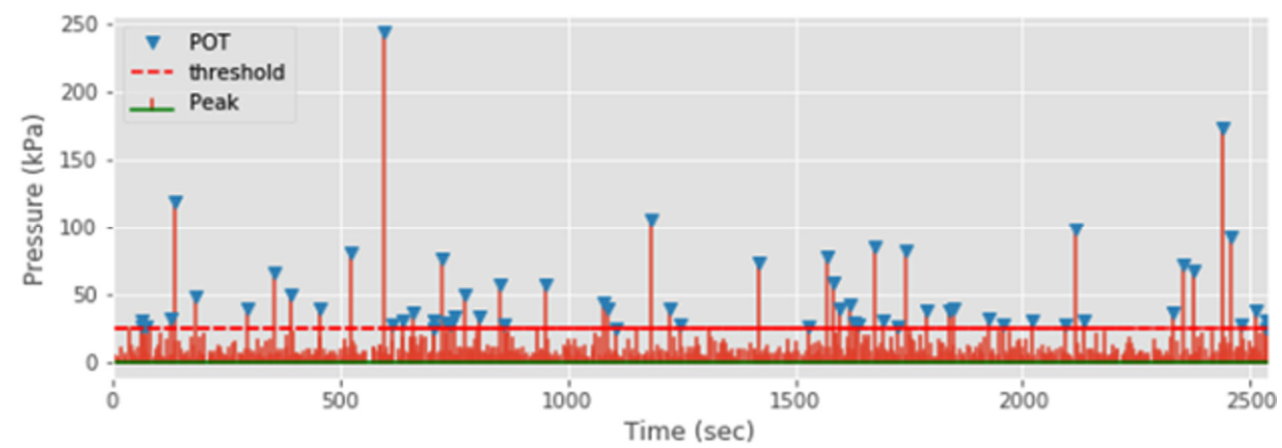

Fig. 562 largest $8 \%$ sloshing peak pressures $(u=24.5 \mathrm{kPa})$ 


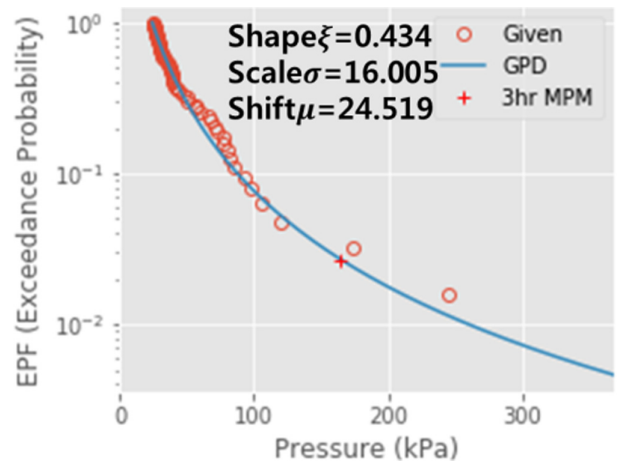

Fig. 6 GPD fitting with largest $8 \%$ sloshing peak pressures $\left(N_{p}=\right.$ $62, I Q R=4.172, \gamma=7.57)$

plot), one can say that all 764 data points are not IID. Hence, the $3 \mathrm{~h}$ MPM of $143.6 \mathrm{kPa}$ is unconvincing.

Next, the other conventional procedure is GPD fitting for the largest $8 \%$ of the data as a rule of thumb, as shown in Fig. 5 and Fig. 6, with a GPD threshold $u=24.3 \mathrm{kPa}$ and $N_{p}=62$. The estimated 3 $\mathrm{h}$ MPM is $164.7 \mathrm{kPa}$, which is larger than $143.6 \mathrm{kPa}$, as shown in Fig. 2. The fitting results look better than Fig. 2. In fact, considering $I Q R=4.172$ and $\gamma=7.57$, Fig. 6 has no outlier. Furthermore, the data follows a Fréchet distribution rather than a Weibull distribution because positive shape parameter $\xi=0.434$. Because this high threshold reduces the bias and the number of data points but increases the variance, it is necessary to choose a lower threshold as far as possible to increase the amount of data and decrease the variance.

Fig. 7 and Fig. 8 are used to check the IID and goodness of fit, and show somewhat nice fitting with $R=0.993$ in Fig. 8 .

The MEF plot shown in Fig. 9 indicates that selecting a threshold of $24.5 \mathrm{kPa}$ is not bad, because the MEF shows a linear tendency above the threshold (see section 2.1) except for a high threshold due
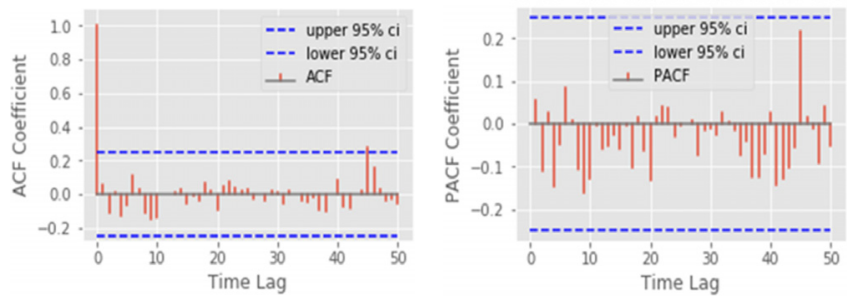

Fig. 7 ACF (left) and PACF (right) for largest $8 \%$ of data $\left(N_{p}=\right.$ $62, u=24.5 \mathrm{kPa})$
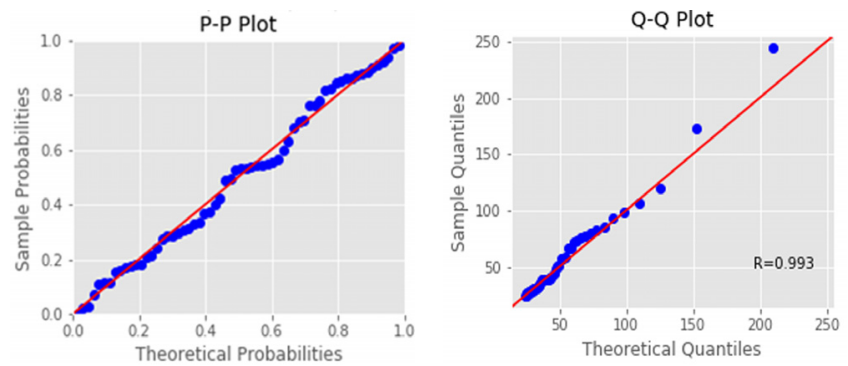

Fig. 8 P-P (left) and Q-Q (right) plots for largest $8 \%$ of data

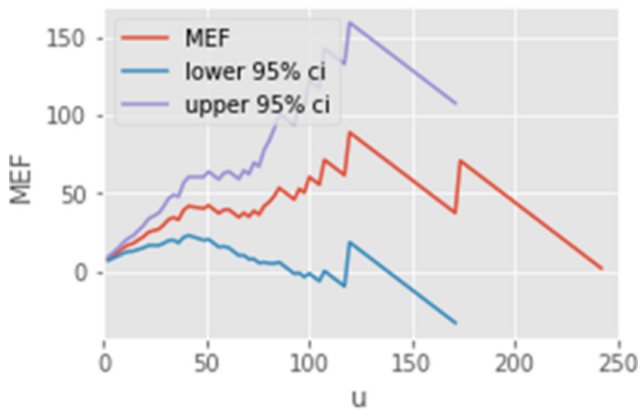

Fig. 9 MEF plot for all 764 data points

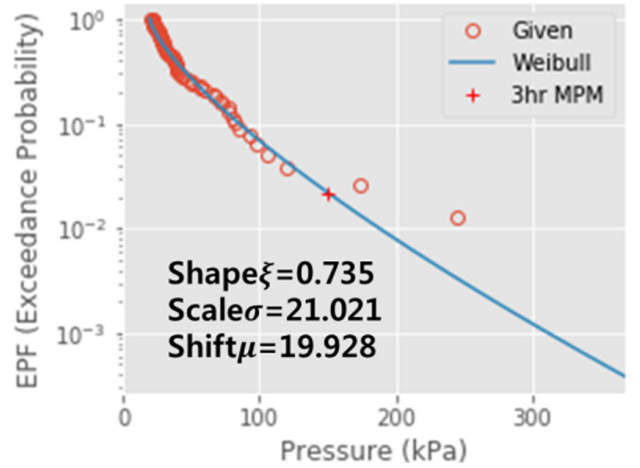

Fig. 10 3-parameter Weibull fitting with largest $10 \%$ of data $\left(N_{p}=\right.$ $77, I Q R=14.738, \gamma=3.58)$

to the lack of data. Hence, it can be said that a proper threshold is around $24.5 \mathrm{kPa}$.

Finally, considering an industry practice of 3-parameter Weibull fitting for the largest $10 \%$ of the data, the $3 \mathrm{~h} \mathrm{MPM}$ of $150.7 \mathrm{kPa}$ is obtained with $u=21.0 \mathrm{kPa}$ and $N_{p}=77$, as shown in Fig. 10. This is slightly larger than $143.6 \mathrm{kPa}$ for the 3-parameter Weibull fitting with all 764 data points in Fig. 2. The last two large pressures are considered the outliers, with $I Q R=14.738$ and $\gamma=3.58$. Considering $\xi>0$ in Fig. 6, the Weibull with MOM is not an appropriate distribution in this case.

\section{GEV and GPD for IID Variables}

Because the data for GEV and GPD should be IID random variables, the block maxima for GEV and the exceedances over the threshold for GPD are used to check IID using the PACF and MEF in this study, respectively. For MEF in Fig. 9, it is, however, somewhat challenging to interpret for a linear region to choose the threshold and disadvantageous to eliminate the manual work for determining the threshold. Hence, PACF is also used in checking IID for GPD in this case.

At first, for GPD, Fig. 7 and Fig. 11 show ACF and PACF for the largest $8 \%, 10 \%$, and $11 \%$ of the data, respectively. All of the PACFs satisfy the $95 \% C I$, Hence, all of the cases pass the IID checks. The $11 \%$ case has the largest number of data points $\left(N_{p}=\right.$ 85 ) among them, and data larger than $11 \%$ cannot pass the IID check. 

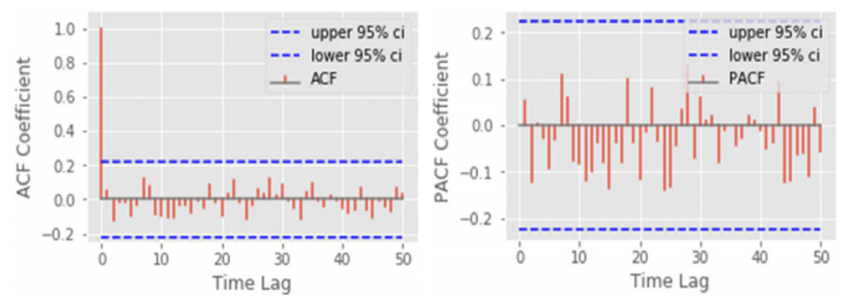

(a) largest $10 \%$ of data $\left(N_{p}=77, u=21.2 \mathrm{kPa}\right)$
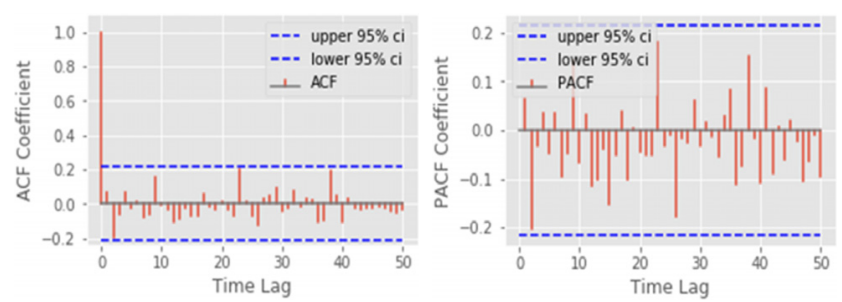

(b) largest $11 \%$ of data $\left(N_{p}=85, u=19.8 \mathrm{kPa}\right)$

Fig. 11 ACF (left) and PACF (right) for largest $10 \%$ and $11 \%$ of data

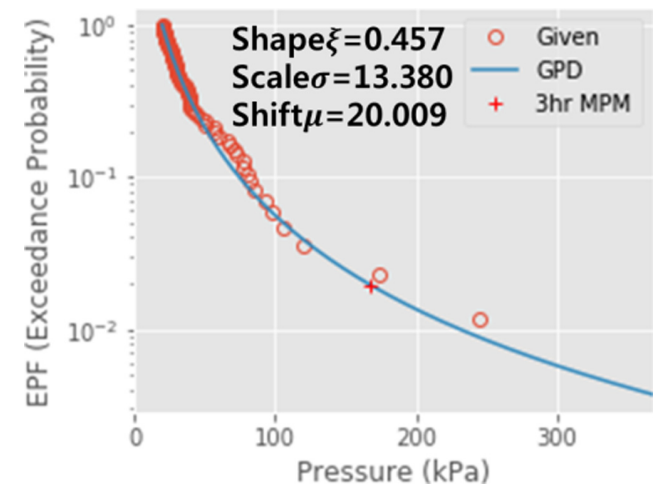

Fig. 12 GPD fitting with largest $11 \%\left(N_{p}=85, I Q R=4.504, \gamma=\right.$ $5.85)$

Therefore, $u=19.8 \mathrm{kPa}$ for the $11 \%$ case is finally chosen for the GPD threshold.

Fig. 12 shows the GPD fitting with the largest $11 \%$ of the data $\left(N_{p}=85\right)$. The estimated $3 \mathrm{~h}$ MPM for GPD is $167.14 \mathrm{kPa}$. This figure also has no outlier with $I Q R=4.504$ and $\gamma=5.85$.

Next, for GEV, Fig. 13 and Fig. 14 show the PACFs and Q-Q plots for block sizes of 4, 6, and 10, respectively. From PACF, all of the cases pass the IID checks. However, the Q-Q plots show that the case of a block size of 10 is the most appropriate choice with the largest $R=0.995$. Hence, the final block size of this study is 10 , with a time window of $2.0 \mathrm{~s}$.

Fig. 15 shows the GEV fitting with 77 tail data points for a block size of 10 . The estimated $3 \mathrm{~h}$ MPM for GEV is $184.8 \mathrm{kPa}$. This figure also has no outlier with $I Q R=0.087$ and $\gamma=200$.

From the GPD and GEV fitting figures, it can be said that these fitting methods with IID checking may give statistically more appropriate design pressures. The positive GEV shape parameter $(\xi>0.489$ in Fig. 15) yields the Fréchet distribution.
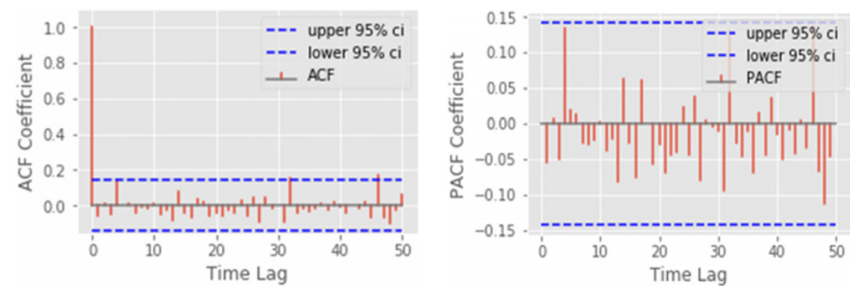

(a) 4 blocks $(0.8 \mathrm{~s})\left(N_{p}=191\right)$
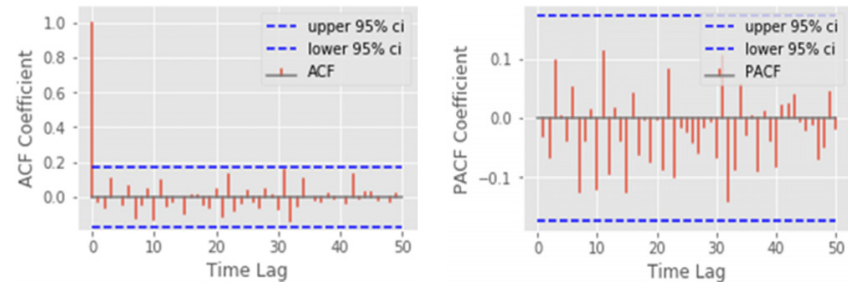

(b) 6 blocks $(1.8 \mathrm{~s})\left(N_{p}=128\right)$
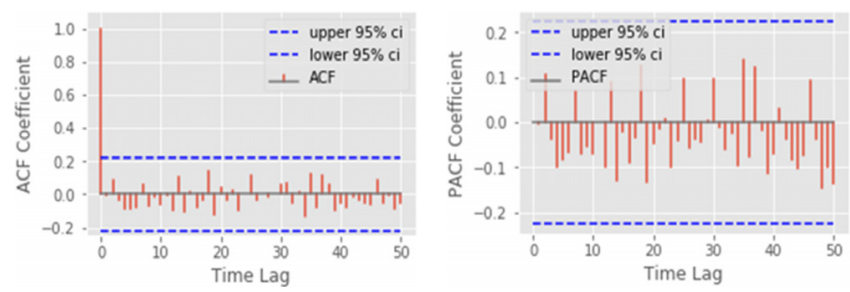

(c) 10 blocks $(2.0 \mathrm{~s})\left(N_{p}=77\right)$

Fig. 13 ACF (left) and PACF (right) for various blocks
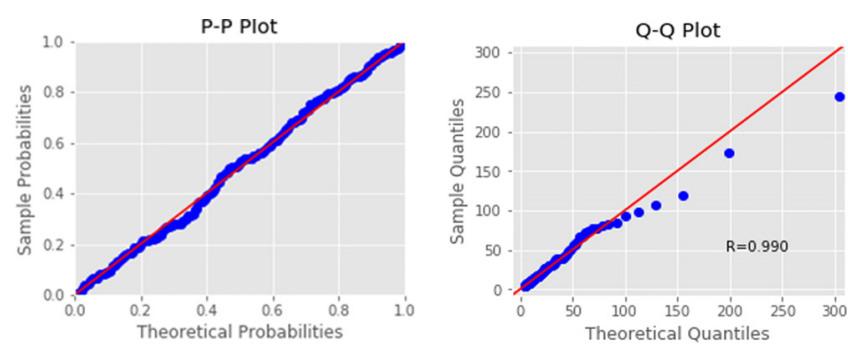

(a) 4 blocks $(0.8 \mathrm{~s})\left(N_{p}=191\right)$
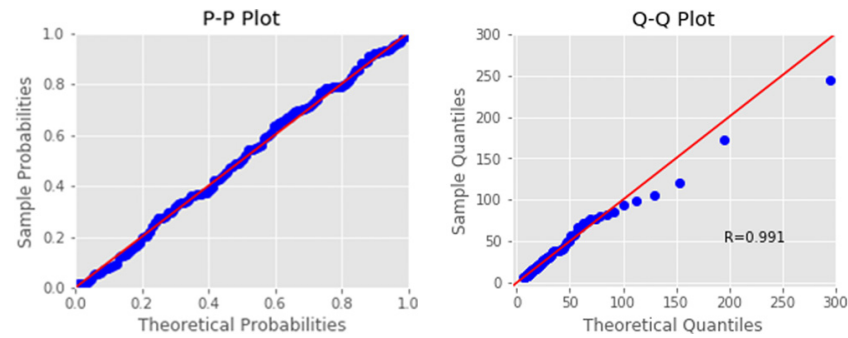

(b) 6 blocks $(1.8 \mathrm{~s})\left(N_{p}=128\right)$
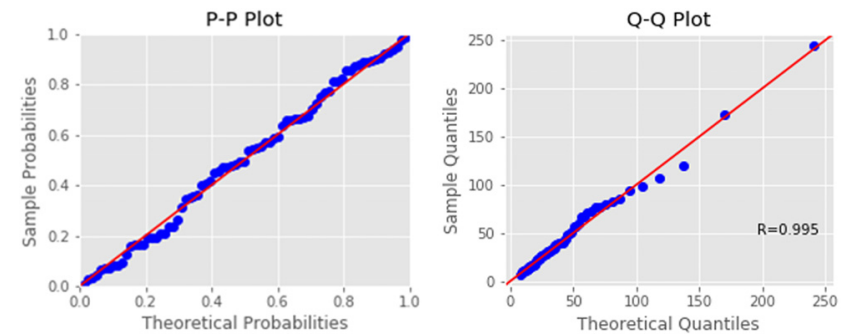

(c) 10 blocks $(2.0 \mathrm{~s})\left(N_{p}=77\right)$

Fig. 14 P-P (left) and Q-Q (right) plots for various block sizes 


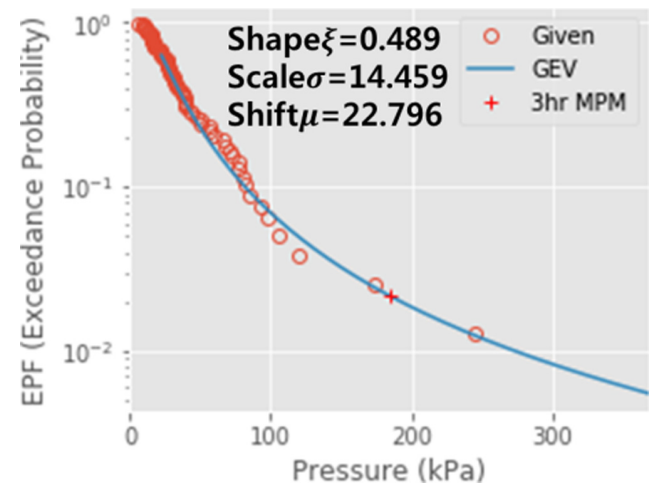

Fig. 15 GEV fittings for block size of $10(I Q R=0.087, \gamma=200)$

\section{Concluding Remarks}

In this study, GEV and GPD were used to fit IID random variables from a sloshing model test, as well as the conventional analysis procedures. In order to apply GEV and GPD, the IID check was carried out in advance for highly nonlinear data by MEF and graphical diagnostic plots. For the feasibility study of the resampling IID variables approach, only one $5 \mathrm{~h}$ sample of sloshing test results was used in this study. Hence, further study with various samples with test durations of $5 \mathrm{~h}$ or longer (e.g., $30 \mathrm{~h}$ ) and other filling cases (e.g., $15 \%$ to $70 \%$ of tank height) may be needed to verify this approach. The following concluding remarks can be made for this study.

(1) In the case of impact pressures obtained by the sloshing model test in this study, the 3-parameter Weibull with MOM may not be an appropriate distribution for all of the sampling data using the conventional procedure with an initial threshold and time window.

(2) The GPD fitting for the largest $8 \%$ of the data, as one of the conventional procedures, gives a relatively reasonable design pressure. Because this high threshold reduces the bias and the number of data points but increases the variance, it is necessary to choose a lower threshold as far as possible to increase the number of data points and decrease the variance. In addition, the obtained positive GPD and GEV shape parameters give evidence that the data to be analyzed follows a Fréchet distribution rather than a Weibull distribution.

(3) The 3-parameter Weibull fitting for the largest $10 \%$ of the data as an industry practice showed a bad performance because of the positive GPD shape parameter for the sample in this study.

(4) Resampling of the IID variables was carried out using ACF, PACF, MEF, and graphical diagnostic plots for GEV and GPD. From this study, resampling IID variables may reduce the number of outliers, and yield a statistically more appropriate design value because of the independent sample.

\section{References}

Balkema, A., de Haan, L., 1974. Residual Life Time at Great Age. The Annals of Probability, 2(5), 792-804.

Bommier, E., 2014. Peaks-over-threshold Modeling of Environmental Data. Project Report 2014:33, Department of Mathematics, Uppsala University. DiVA, id: diva2:760802.

Choi, Y.H., 2016. Extreme Value Analysis of Environmental Data. Proceedings of the Fall Conference of the Korean Society of Ocean Engineers, 181-186.

DNVGL, 2015. Position Mooring. DNVGL-OS-E301.

DNVGL, 2017. Environmental Conditions and Environmental loads. DNVGL-RP-C205.

Fillon, B., Diebold, L., Henry, J., Derbanne, Q., Baudin, E., Parmentier, G., 2011. Statistical Post-Processing of LongDuration Sloshing Test. ISOPE-I-11-526.

Fisher, R.A., Tippett, L.H.C., 1928. Limiting Forms of the Frequency Distribution of the Largest and Smallest Member of a Sample. Mathematical Proceedings of the Cambridge Philosophical Society. 24(2), 180-190.

Gnedenko, B.V., 1943. Sur La Distribution Limite Du Terme Maximum D'Une Série Aléatoire. Annals of Mathematics, 44(3), 423-453.

Gumbel, E.J., 1954. Statistics of Extremes. Dover, Mineola, New York, ISBN 0-486-43604-7.

Smith, RL, 1985. Maximum Likelihood Estimation in a Class of Nonregular Cases. Biometrika, 72(1), 67-90.

Kim, S.Y., Song, J., 2012. Estimation of Car Insurance Loss Ratio Using the Peaks over Threshold Method. Korean Journal of Applied Statistic, 25(1), 101-114. https://doi.org/10.5351/KJAS. 2012.25.1.101

Kim, S., 2017. Development of Long-term Prediction Procedure and Outlier Analysis for Sloshing Impact Loads on LNG Cargo Tank. Doctoral Thesis. Seoul National University.

ITTC, 2017. ITTC Quality System Manual - Recommended Procedures and Guidelines - Procedure - Sloshing Model Tests. 7.5-02-07-02.7.

Ochi, M.K., 1990. Applied Probability and Stochastic Processes - In Engineering and Physical Sciences. John Wiley \& Sons, ISBN-10: 0471857424, ISBN-13: 9780471857426.

Pickands, J., 1975. Statistical Inference Using Extreme Order Statistics. The Annals of Statistics, 3(1), 119-131.

Tukey, J.W., 1977, Exploratory Data Analysis. Addison-Wesley Publishing Company, 1st Edition, ISBN-10: 0201076160, ISBN-13: 978-0201076165.

Xu, J., Long J.S., 2005. Using the Delta Method to Construct Confidence Intervals for Predicted Probabilities, Rates, and Discrete Changes. Lecture Notes, Indiana University. 